\title{
Fish stock assessments and predictions: integrating relevant knowledge. An overview*
}

\author{
ØYVIND ULLTANG \\ Department of Fisheries and Marine Biology, Bergen High Technology Center, University of Bergen, N-5020 Bergen, \\ Norway. E-mail: Oyvind.Ulltang@ifm.uib.no
}

\begin{abstract}
SUMMARY: A variety of existing scientific knowledge can be better utilised for improving stock assessments and predictions. In particular, failure to take account of environmental effects, including both biotic and abiotic factors in the term environment, is a serious source of error in fish stock assessments and limits the time horizon of reliable predictions. The report of the EU-funded SAP project and a set of papers resulting from communications presented at the SAP symposium in Bergen in December 2000 emphasis the potential gains by integrating relevant knowledge of processes affecting fish stocks.
\end{abstract}

Key words: stock assessments, predictions, environment.

\section{THE SYMPOSIUM}

The SAP Symposium: "Fish Stock Assessments and Predictions: Integrating Relevant Knowledge" was held in Bergen, Norway 4-6 December 2000. It was arranged as a summing up of the findings in the SAP project, but was also intended to be a forum for presentation of similar scientific information by scientists outside the project.

Nearly 150 scientists from Europe, North America, Africa and Asia attended the symposium. Altogether 47 presentations (39 oral and 8 poster presentations) were given during the symposium sessions. A subset of these was submitted for inclusion in this volume of Scientia Marina, and 27 papers were finally accepted after peer review.

This overview is based on the SAP project report (Ulltang and Blom, 2001), oral presentations at the symposium and papers included in this volume.

\footnotetext{
*Received May 27, 2002. Accepted July 8, 2002.
}

\section{THE SAP PROJECT}

The main objective of the SAP project (EU-FAIR CT 97-3805, 1998-2001) was to advise on how existing scientific knowledge can be better utilised for reducing the uncertainties and increasing the time horizon of fish stock predictions.

The following partial objectives were defined:

- Identify main causes of shortcomings in present fish stock assessments and predictions (Task 1).

- Consider the limits set by nature on predictability of fish stock development (Task 2).

- Review "the state of the art" within relevant research areas and identify the kind of scientific knowledge potentially useful in fish stock predictions which exists without being effectively used at present. Discuss how such knowledge most effectively could be used and propose future coordinated research for expanding the scientific knowledge relevant for fish stock assessment (Task 3). 
- Carry out case studies for selected areas and stocks with the aim of demonstrating the potential for improvements in quality and time horizon of fish stock predictions (Task 4).

- Evaluate how communication/co-operation between fish stock assessment experts and experts within various fields of basic marine sciences can be improved (Task 5).

The following four major geographical areas were considered:

Area 1: Norwegian and Barents Seas and Icelandic waters

Area 2: North Sea, Skagerrak/Kattegat and Baltic

Area 3: Mediterranean

Area 4: Areas west of the Iberian Peninsula

The main focus of the project was on how predictions can be improved by utilising knowledge/understanding of how a fluctuating biotic and abiotic environment influences fish stocks. The complexity of this task is rooted in the large (infinite) number of processes affecting the stocks. We observe and try to predict the combined effects of the various processes/factors. Progress in science depends partly on our ability to separate the various effects and evaluate specific relationships based on observations where effects of other factors can be isolated or assumed to be minimal. The challenge is to identify the main processes/factors and quantify their effects on stock parameters (recruitment, growth, mortality). If possible we should also develop methods for predicting key environmental variables.

\section{CAUSES FOR SHORTCOMINGS}

Causes for shortcomings in present fish stock assessments and predictions include problems with stock units; catch statistics, including species and age/size composition of catches; fishery independent assessment data; biological, including life history data; internal (density/stock size dependent) effects; multispecies effects; environmental effects; and population and assessment models. Underreporting of catches, misreporting by stock areas or species and the unknown amount of discards is a problem for many stocks/areas, introducing errors in the VPA-calculated time series of recruitment, fishing mortalities, total stock biomasses and spawning stock biomasses. Multispecies effects may be considered as part of environmental effects, including both biotic and abiotic factors in the term environ- ment. Influences from the environment are in general not taken into account in the assessments, and it was concluded in the project that failure to take account of environmental effects was a serious source of assessment error in all areas. For many stocks, lack of information about environmental conditions, or lack of use of relationships between stock parameters (variables) and environmental conditions, is also a main factor limiting the time horizon of predictions.

Concentration on mathematical model development may have drawn attention from the all-important problem of collecting and analysing appropriate data to get a better insight into basic population dynamics. Only concentrating on modelling is selfdefeating because the performance of even the best model is constrained by the quality of the data and the understanding of basic processes. More effort should be devoted to biological understanding of the resources and a better understanding of the dynamics of the fishery by those involved in fisheries management (Hilborn, these proceedings). The biological environment, and in particular interactions between fish species, is sometimes modelled explicitly when predictions of fish stocks are made. However, the traditional single-species age-structured stock assessment models ignore biological and environmental effects, and assumptions about future rates of growth, mortality and recruitment used in predictions are still most often based on some statistical treatment of past values and rarely include environmental information. We need different kinds of predictions as well as better predictions (Brander, these proceedings). John Pope (oral symposium presentation) noted that basic biological knowledge should be better utilised by ICES assessment working groups, and he also suggested that too much effort is going into short-term forecasts compared to long-term trends (effects). Environmental effects may alter the spatial distribution of a stock, leading to possible changes in catchabilities in both fisheries and surveys. Marchal et al. (these proceedings) present trends in catchability in a number of North Sea demersal fisheries. They conclude that taking account of time variation in catchability would result in some improvement in the performance of traditional stock assessments and new perceptions of historical trends of biomass and fishing mortality.

For many stocks long time series of catches indicate substantial long term fluctuations as shown for blue fin tuna in the Northeast Atlantic and Mediterranean Seas (Fromentin, these proceedings). Study 
of catch variability of a large number of stocks over different time periods indicates red noise (variance increasing with increased time period) in these sequences (see SAP project report, part II). Thus, fishery managers and scientists are confronted with variables that do not explore their size limits quickly but rather wander and reach new extreme values continually. Such a pattern in fish catch variability suggests that there is no equilibrium yield, the latter being the basis of most models presently used for fisheries management; steady yield seems to be the exception rather than the rule.

The assessment and management of Mediterranean fisheries faces some significant challenging problems as described by Lleonart and Maynou (these proceedings): no TAC or adaptive management is in place. There is a lack of feedback among the three agents for an adaptive management (administration, fishermen and scientists). The social pressure on the administration regarding needs for assessment is almost null. Acquisition of data suffers from the lack of interest of all sectors involved. Actions to remedy this situation are discussed by the authors and in the SAP project report.

\section{LIMITS ON PREDICTABILITY}

In the project, we distinguished between two kinds of uncertainty: objective uncertainty arising from the underlying variability in stochastic processes (variability), and subjective uncertainty due to incomplete knowledge of the system (ignorance). The objective uncertainty puts limits on predictability, while the subjective uncertainty can be reduced by increasing the accuracy of observations and integrating more knowledge in the management advice process. Task 2 addressed the variability question, while task 3 dealt with how we can reduce the subjective uncertainty by expanding and integrating relevant knowledge into stock assessments and predictions. However, these tasks were inter-linked.

It was noted that there is no, and cannot be any, clear answer to the question of what are the limits set by nature on predictability. But from our present knowledge of the different processes and on-going research, we can identify physical and biological processes where variability sets limits on predictability. At the same time, we can identify processes where there are good prospects of explaining more of the observed variability by reducing our ignorance.
In considering how knowledge of interactions with the environment can be utilised in stock predictions, a key question is of course whether the abiotic environment is predictable. Environmental conditions that can be measured at the time of the assessment, influencing biological input data, can improve short-term stock predictions. However, if environmental development could be predicted, this could further improve the short-term predictions and in particular improve stock predictions on a longer time scale. In all the regions considered in the project there have been documented ecosystem effects of the North Atlantic Oscillation (NAO). But is the NAO index itself predictable? At present the answer is no. We may, however, be able to give some statements that may aid management and/or assessment in general terms and in the medium term (1-5 years, say). This possibility arises because the NAO has shown a decadal time-scale variation giving, perhaps, a better than even chance of guessing the sign of the process. However, this is liable to fail spectacularly in individual years.

Climate prediction is at a rather early stage. However, as noted by Brander (these proceedings), an immense scientific effort is now going into modelling likely future climate scenarios. As noted by Mojib Latif (guest lecturer at the $3^{\text {rd }}$ SAP meeting, Hamburg, April 1999), during the next 20 years there will probably be huge improvements in the coupled ocean-atmosphere models used for climate predictions. The El-Niño/Southern Oscillation phenomenon (ENSO) is the prototype for climate prediction, and has been paid much attention in the research. Temperature anomalies in the Atlantic Ocean, starting off the coast of Florida, have a cycle of 10 years, and can be predicted by the coupled ocean-atmosphere circulation model. Also, strong El Niños can be traced in the climate of the Atlantic Ocean, and there seems to be a predictive pattern in the climate of the North Atlantic Ocean coupled with variations in the North Atlantic Oscillation (NAO).

\section{SCIENTIFIC KNOWLEDGE POTENTIALLY USEFUL IN FISH STOCK PREDICTIONS}

Short-term predictions (for a definition of shortterm prediction based on life expectancy, see Brander, these proceedings) can be based on estimates of fish alive now and on recently observed rates of growth, recruitment and mortality, although interac- 
tions with the environment affecting growth and mortality in the short term should be included. For long-term predictions, it is not sensible to use the past record of rates of growth and recruitment on its own as the basis for predicting future rates. The fact that it is difficult to establish the processes by which the environment affects variability in rates of growth, recruitment and mortality has of course held back the application of environmental data in predictions. The need to put more effort into this area is becoming more urgent as it becomes increasingly apparent that substantial climate changes are likely over the time periods covered by medium and longterm predictions.

Köster, Schnack and Möllmann (these proceedings) give examples of knowledge of biological processes that can be utilised to improve short- and long-term predictions, including habitat suitability, fish condition, predation mortality, fecundity, quality of spawn, distribution of spawn, egg and larval survival and cannibalism. They note that medium- to long-term predictions depend heavily on the recruitment models employed. When major changes in the environment are likely to happen in a certain area, the impacts have to be incorporated into stock-recruitment models. For this purpose, relevant scenarios of environmental conditions and proxies to identify the state of the system have to be developed. Besides the explanatory power of relevant environmental variables, their predictability and related predictive time frames are of major importance for a potential application in stock predictions. Svein Sundby (oral symposium presentation) discussed the relations between physical environment, plankton and fish and compared the seasonal and inter-annual variability of environmental factors most relevant for larval fish development (temperature, zooplankton, wind and light). Temperature is highly correlated with other variables. In the northern areas, zooplankton biomass shows region-specific temporal trends with a difference between Norwegian Sea and Barents Sea and varies between cold, average and warm years (increase with temperature). For realistically describing growth, survival and condition of larvae in different physical environments it is necessary to apply individual based models.

Temperature was identified as a simple and an easily measured key parameter with a profound influence through all trophic levels in the regions of the North Atlantic ecosystem. Temperature has a complex linkage to recruitment and growth in fish stocks because 1) it partly influences growth rates in fish stocks directly, 2) it partly influences fish stocks through its influences on lower and higher trophic levels and 3) it acts as a proxy for other ocean climate parameters like wind mixing, advection and light conditions.

The strongest response of fish stocks to temperature changes has been documented in the northern areas, i.e. in the Barents Sea. This probably has three main reasons. Firstly, the changes in vital rates per degree Celsius for most fish stocks is much larger at the lower temperature range; secondly, at the lower range of temperature most marine organisms throughout the trophic levels of the ecosystem respond uniformly with increasing vital rates with increasing temperature resulting in a general increase in the transfer of production through the tropic levels of the ecosystem. Thirdly, the interannual fluctuations of temperatures are weaker in the southernmost part of the areas considered, i.e. the Mediterranean Sea.

Forcing functions can vary greatly in time and place. Basically, these result from coupling between the ocean and atmosphere. In most of the local regions in the North Atlantic advection of specific water masses from outside is a major forcing function and has been reported to influence ecosystem features, thermal structure and fish stock productivity. The most obvious climatic effects on the ocean of a high NAO index are increased wind mixing in the Northeast Atlantic, changes in advection of water masses, changes in the winter sea temperature, and changes in the hydrologic conditions (anomalous wet conditions in the northern areas and anomalous dry conditions in the southern areas).

There are great differences between the various regions/areas and stocks in the mechanisms and in the knowledge of mechanisms involved in environmental impacts on fish stock development. In some cases there are good correlations between environmental parameters and the development in fish stocks, but the actual mechanisms which cause the stocks to fluctuate are largely unknown. For other stocks and/or areas the mechanisms are known, at least to some extent, so correlations can be supported and explained by "cause and effect" considerations. Establishment of relationships between environmental data and fish stock characteristics or parameters as well as the incorporation of such relationships into assessments should be based upon a good understanding of the ecology of the particular system or region and allow some understanding of the mechanisms underlying the statistical correlations. 
In general, long time series of phytoplankton, zooplankton and other variables, including physical variables, exist for various locations in all project regions. For the Barents Sea-Norwegian Sea, several relationships have been established between vital rates in the stocks of cod, herring and capelin and time series of environmental variables, and some relationships are now being introduced in stock assessments. High ambient sea temperature favours growth of cod and herring, and environmental conditions (in particular sea temperature in the case of cod and herring) have clear effects on stock-recruitment relationships. There is a close link between length at the 0-group stage and year class strength. Further analyses are presented in the project report: see especially the project case studies for the Barents Sea (SAP project report, part III). For predicting cod recruitment, the analysis by Huse and Ottersen (these proceedings) ranks cod spawning stock biomass as the most important contributor to the output, followed by capelin biomass and sea temperature.

In the Baltic, a time series of a combined variable ("reproductive volume") which represents the volume of water with the minimum salinity and oxygen conditions for successful cod egg hatching has been derived in addition to the many other long time series of environmental variables (Köster, Schnack and Möllmann, these proceedings; Köster et al., these proceedings). The reproductive volume is linked to large-scale atmospheric processes determining inflow of saline, oxygenated water from the North Sea. For both sprat and cod in the Baltic, it is shown that relating data for subsequent early life history stages, i.e. potential egg production, realised and surviving egg production as well as larval and 0group abundance, allows the identification of critical periods within the recruitment process of both species. Environmental variables having a significant influence on the survival at critical life stages can then be incorporated into modified stockrecruitment models. For the Belt Sea cod, Oeberst and Bleil (these proceedings) show that a trawl survey during the beginning of the spawning season to estimate the portions of active female spawners can be used for assessing the actual potential population fecundity and reaching a first estimate of year class strength.

For the North Sea area, relationships were reported between different environmental parameters and sandeel. For cod, the use of environmental information to improve stock predictions has advanced in recent years. The most studied environmental factor that influences North Sea cod is the sea surface temperature (SST). In particular, temperature has been related to recruitment (negative correlation, see e.g. Lindley et al., these proceedings) and growth (Brander, these proceedings), and statistical short-term forecasts of temperature may be used in short-term forecasts of recruitment and thereby extend the time horizon of recruitment predictions. The reliability of such predictions is still low, but the approach should be pursued (Planque et al., these proceedings).

In the Irish Sea, recruitment of cod and plaice appears to be related to sea temperature during winter-spring. At present the temperature signal is the best single environmental proxy to explain the variability in the recruitment of Irish Sea cod, low temperature in winter-spring being associated with "good" environmental conditions (see SAP project report, part II).

Borges et al. (these proceedings) investigate the effect of wind conditions and NAO on sardine yields in the Portuguese fishery. They conclude that recruitment is low when the frequency and intensity of northerly winds in winter exceeds a certain limit. The mechanism is presumably that northerly winds increase upwelling during the sardine winter spawning season, strong upwelling being unfavourable for recruitment. Northerly winds are associated with positive NAO anomalies. Carrera and Porteiro (these proceedings) explain the failure of sardine catches off Galicia (NW Spain) by stock depletion caused by reduced recruitment and increased fishing mortality. The depletion affects both the feeding migration of adult fish and the recruitment occurrence off South Galicia. They further note that the recruitment success may depend on the size of the spawning area: the more coastal the area the less important the influence of the oceanographic events will be.

In the Mediterranean Sea the influence of local environment (e.g. river runoff and winds) on the productivity is becoming more apparent as a consequence of new investigations being carried out, and it is now possible to describe favourable and unfavourable environmental conditions for productivity of some stocks and to predict their future recruitment in terms of expected above or below normal levels of offspring. Levi et al. (these proceedings) consider SST as a proxy for oceanographic processes affecting recruitment of red mullet in the Strait of Sicily, and incorporate SST in stockrecruitment models. For a given spawning stock 
level, higher recruitment levels correspond to higher than average SST during early life stages, the mechanism probably being that high SST is associated with reduced upwelling and a lower level of offshore transport.

In summary, two important points emerged from the project case studies and symposium presentations:

- Climate is important, and its signals can be recognised in stock records and recruitment time series.

- These signals can be inserted into stock-recruitment models and can improve their performance, as shown for cod, herring and capelin in the Barents Sea, Irish Sea cod, Baltic cod and sprat, and red mullet in the Strait of Sicily.

The use of long time series was addressed in several contributions. Nils C. Stenseth and co-workers (oral symposium presentation) presented a work on modelling the dynamics of the Norwegian coastal Skagerrak cod population based on long-term monitoring data (annual surveys since 1919). It was shown that the stochastic component of stock fluctuations may lead to longer-term fluctuations superimposed upon short-term periodic fluctuations through resonance. During the discussion the importance of continuing long time series was stressed.

\section{MODELLING APPROACHES}

Both the biotic and abiotic environment in which fish stocks exist affect their population dynamic processes through numerous pathways. This may argue for capturing these processes in a rather complex mathematical model. The various types of population dynamics models can be broadly classified according to their level of complexity, ranging from simple stock production models to the more complex models which incorporate age, size, species and area (possibly further including sex and maturity).

The purposes of models in the management context are:

- to interpret the past history of an exploited stock to quantify factors of importance to management and to determine the present stock status.

- to predict possible long-term effects of management actions (e.g. effects of effort management on long-term yield)

- to estimate short/medium-term measures that will bring the stock status to a predefined, desired level.
Complex models (e.g. highly disaggregated multispecies models) may be useful for point (i) and possibly also point (iii). It was noted in the project that some management measures could be more directly affected by environmental changes than others (examples of those probably most affected are temporal closures, spatial closures and protected areas). Conventional scientific input to management is based on spatially homogeneous models in which the environment is assumed to be constant, and in which the individuals comprising the target populations all respond to external factors independently of each other. However, these highly integrated procedures do not allow the modeller to take into account features of population dynamics and interactions with the environment which have become recognised in recent decades, and which may be of great importance for some stocks in some circumstances.

However, there may be several drawbacks to complexity, and simpler models that capture the main issues may be better for long-term predictions since these must integrate many factors which we do not understand very well. Production models are an example of a simple form of predictive model. It is, however, important to stress that while simple models that broadly summarise our knowledge might be preferred to more complex models for prediction and for management purposes they typically require the backing of more complex models, data sets and studies to guide their formulation. The task of providing better ways of explaining complex results to managers is discussed in the project report using simplified models. In the case studies for the Norwegian and Barents Seas (see SAP project report, part III) it was considered how age-structured models may be reduced to bulk biomass models for prediction purposes using the Northeast Arctic cod as an example.

Several symposium contributions compared different models or presented results of using new modelling approaches. Abaunza et al. (these proceedings) compare results of biomass dynamic models applied to the southern horse mackerel stock with results of VPA-based methods. In the discussion at the symposium it was noted that it might not be a question of using one type of model or the other. Age-based models could be used for interpreting the past and biomass models for predictions. Stergiou et al. (these proceedings) apply various time series models to the swordfish catches in the eastern Mediterranean and conclude that the X-11 census technique outperforms the other methods 
applied both in terms of fitting and forecasting performance. Bjarte Bogstad and Kristin G. Frøysa (oral symposium presentation) presented Fleksibest - an age-length structured assessment model - as an alternative to XSA for Northeast Arctic cod. It is easy to incorporate various biological processes in the model. The length structure makes it possible to estimate how the exploitation pattern varies with the growth of the cod. Also, the assumptions used in Fleksibest are more transparent than the assumptions in more traditional models. Björnsson and Sigurdsson (these proceedings) demonstrate the use of the Bormicon model on a stock of golden redfish partly based upon catch at length data with some age data. The strength of this method is that it can make analyses for periods for which only catch at length data exist. Huse and Ottersen (these proceedings) present an artificial neural network (ANN) approach for predicting biomass (1, 2 and 3 years ahead) and recruitment (3 years ahead) of Northeast Arctic cod, illustrating the strong ability of ANN models as a forecast technology. The authors note that a potential disadvantage of the approach is that we do not achieve an understanding of the mechanisms causing the specific relationship between the variables. To provide a better understanding there is a need for models that build on evolutionary and ecological principles.

\section{IMPLICATIONS FOR FISHERY MANAGEMENT}

With respect to fishery management, a key question is whether we can discriminate between the various effects of a fluctuating biotic and abiotic environment and the effects of the fishery itself on a stock. When a stock collapses, it is typically asked whether this was caused by the fishery or the environment. This is the wrong question to ask. Through evolutionary processes, fish stocks adapt to a fluctuating environment. The problem we should address is how the fishery (and other activities by man) may perturb this adaptation. For example, if the reproductive strategy of a stock is linked to periodicities in fluctuations in the abiotic environment through evolutionary processes, intensive exploitation changing the population's demographic and genetic structure and vital rates could have drastic consequences such as those discussed for Northeast Arctic cod (Øyvind Ulltang, oral symposium presentation). We should try to predict these consequences through simulation studies, linking hypotheses on stock dynamics and effects of environmental fluctuations at the different life stages.

In addition to generally increasing the reliability of short, medium and long term predictions and thus give a sounder basis for fishery management, introduction of environmental effects in population models will enhance the possibilities to study the combined effects of environmental variability and exploitation on stock abundance. Reference points, stated in terms of fishing mortality rates or biomass, or in other units, are a key concept in implementing management plans consistent with a precautionary approach to fishery management. Incorporating environmental and biological information such as multispecies effects into population models can improve the definition and identification of appropriate reference points.

The preparation of short-term predictions is a major, costly and time-consuming part of annual stock assessments. Since resources in fisheries assessment are finite, the process of carrying out such predictions is to some degree in conflict with the work needed for medium and long-term prediction. The structure of models and kind of information used are different. Dynamics in medium and long time scales is dominated by variability in recruitment and possibly also growth and mortality. Regular annual short-term predictions do not lead to better medium and long-term predictions, and it was suggested that too much effort is going into shortterm forecasts compared to assessing long-term trends. Kell et al. (these proceedings), presenting a work on management strategies for Atlantic tuna stocks, show that initial depletion level and reference points chosen for management matter more than assessment frequency.

\section{THE WAY FURTHER}

Failure to take account of environmental effects, including both biotic and abiotic factors in the term environment, is a serious source of error in fish stock assessments and limits the time horizon of reliable predictions. Based on the outcome of the SAP project and these proceedings, research priorities to remedy this situation can be summarised as follows:

To facilitate the use of environmental data within an ecosystem approach to fisheries management, time series of environmental life history indices relating to each age group (stage) of fish should, where possible, be established for each particular 
stock (or functional group in complex systems). The processes behind correlations between environmental factors and stock parameters, the causal relations and more details of the mechanisms should be explored and further understood, and research on alternative approaches to predicting ocean climate should be continued in order to increase the usefulness of environmental parameters in stock predictions. In particular, further work on explaining and predicting recruitment variability by including environmental signals is required, and there is a need for more focus on predator-prey/multispecies models for explaining/predicting year-to-year variations in fish growth and mortality. Research aimed at elucidating possible genetic selectivity in exploitation and its consequences for a stock's adaptation to a fluctuating environment should be encouraged. Simulations based on realistic structural relationships should be used to assess how environmental information can be used to predict the impact of management actions. Further research on statistical models (e.g. time series analyses) in fisheries management, taking into account environmental parameters and biological data, is encouraged.

It was noted in the proposal for the SAP project that present fish stock assessment practice is characterised by too sharp a de facto separation of fish stock assessment experts and experts within various fields of basic marine sciences. To apply all relevant knowledge in stock assessments, it is important to change this situation both by improving the communication between the groups and by involving experts in the various fields of basic marine sciences in future stock assessments. Ample thought should therefore be given to the question of how to achieve this.

\section{REFERENCE}

Ulltang, Ø. and G. Blom (eds.). - 2001. EU FAIR-CT 97-3805. Sustainable fisheries. How can the scientific basis for fish stock assessments and predictions be improved? (SAP). Final project report, parts I-III. University of Bergen, September 2001. 639 pp. 